\title{
Estudio Retrospectivo de 91 Cirugías de Elevación de Seno Maxilar para Rehabilitación sobre Implantes
}

\author{
Restrospective Research in 91 Maxilary Sinus Floor \\ Elevation Surgery for Implant Rehabilitation \\ Sergio Olate*; Leandro Pozzer ${ }^{* *}$; Aníbal Henrique Barbosa Luna**; \\ Renato Mazonetto*; Marcio de Moraes** \& José Ricardo de Albergaria Barbosa**
}

OLATE, S.; POZZER, L.; LUNA, A. H. B.; MAZONETTO, R.; MORAES, M. \& BARBOSA, J. R. A. Estudio retrospectivo de 91 cirugías de elevación de seno maxilar para rehabilitación sobre implantes. Int. J. Odontostomat., 6(1):81-88, 2012.

RESUMEN: Los injertos óseos para elevación del piso sinusal han sido utilizados por largo tiempo con buenas tazas de éxito. EL objetivo de esta investigación fue estudiar el comportamiento intraoperatorio y postoperatorio de 91 cirugías consecutivas de elevación de seno maxilar. Fueron seleccionados 72 pacientes de entre 18 y 66 años para realizar el procedimiento con anestesia local o general; se estudiaron variables preoperatorias, intraoperatorias, postoperatorias, instalación de implante y complicaciones de la cirugía. El análisis de datos se realizó con la prueba test de Fisher con $p<0,05$ para establecer significancia estadística. En el $74,7 \%$ se utilizó hueso autólogo con o sin ayuda de biomaterial, siendo que en $86,8 \%$ se utilizó un sitio donante intrabucal. Se instalaron 101 implantes siendo un 69,3\% instalado en un segundo procedimiento quirúrgico y los restantes 31 implantes instalados en el mismo tiempo quirúrgico de instalación de injerto óseo; no se observó diferencia estadística entre el éxito de los implantes instalados en uno o dos tiempos quirúrgicos $(p>0,05)$; se observo la pérdida de 4 implantes, los cuales no fueron asociados a ningún tipo de material utilizado para el relleno óseo $(p>0,05)$. La principal complicación fue la laceración de membrana, presente en el 18,7\%, postergándose el procedimiento para ser realizado nuevamente en 13 de los 17 casos. Finalmente se puede concluir que la elevación de seno es un procedimiento reconocido y que es viable de presentar y asegurar estabilidad de los implantes instalados para el desarrollo de la oseintegración.

PALABRAS CLAVE: seno maxilar, implante dental, reconstrucción ósea.

\section{INTRODUCCIÓN}

La reconstrucción ósea para la instalación de implantes ha demostrado altas tazas de éxito (Adell et al., 1981). Diferentes técnicas han sido aplicadas para la reconstrucción ósea, tales como la instalación de injertos en bloque, injertos en partículas con o sin ayuda de malla de titanio, entre otros, mostrando versatilidad y predictibilidad en las técnicas reconstructivas (Breine \& Branemark, 1980).

En maxila posterior, las condiciones anatómicas, la etiología de la pérdida dentaria, el tiempo transcurrido sin dientes posteriores son importantes elementos que determinan la cantidad de reabsorción ósea y las limitaciones para la instalación de implantes (Misch, 1997). En estos casos, una opción es la instalación de implantes cortos (Griffin \& Cheung, 2004), la cual ha presentado buenos resultados en buena parte de los estudios publicados; sin embargo, algunas investigaciones han evidenciado una menor taza de éxito debido a la curva de aprendizaje del cirujano, la calidad ósea, calidad y tipo de implantes y la técnica quirúrgica empleada (Ivanoff et al., 1999; Olate et al., 2010). Por otra parte, la maxila posterior presenta menos hueso cortical (UIm et al., 1999), permitiendo muchas veces la migración de los implantes instalados hacia dentro del seno maxilar (Kluppel et al., 2010). Finalmente, otra opción está en la modificación del hueso con el uso de osteotomos, compactando el hueso del lecho que recibirá el implante (Emmerich et al., 2005).

\footnotetext{
* Unidad de Cirugía Oral y Maxilofacial, Facultad de Medicina, Universidad de La Frontera, Chile

* División de Cirugía Oral y Maxilofacial, Universidad Estadual de Campinas, Campina, Brasil.

${ }^{* * *}$ Departamento de Clínica y Odontología Social, Universidad Federal de Paraíba, João Pessoa, Brasil.
} 
Una de las técnicas aceptadas, debido a sus ventajas, es la instalación de injerto óseo dentro o fuera del seno maxilar para aumentar el volumen óseo que recibirá el implante (Jensen et al., 1994; Lekholm et al., 1999), ya que una de las mas importantes limitaciones anatómicas está en la pneumatización del seno maxilar y la reabsorción ósea del aspecto bucal (Sharan \& Madjar, 2008; Tiwana et al., 2006).

La técnica de elevación de seno maxilar a través de ventana lateral fue desarrollada y publicitada por Boyne \& James (1980) y Tatum (1986) (Fig. 1); actualmente se reconoce esta técnica como segura y eficiente con amplio uso en diferentes condiciones anatómicas (Raja, 2009). Diferentes investigaciones han reportado el éxito de esta técnica (Chiapasco et al., 2008; Wiltfang et al., 2005), señalando que una de las principales complicaciones esta en la ruptura de la membrana sinusal (Viña-Almunia et al., 2009).

El objetivo de esta investigación fue estudiar las condicionantes de la cirugía de elevación de seno maxilar e identificar los resultados postquirúrgicos inmediatos y mediatos en 91 cirugías consecutivas

\section{MATERIAL Y MÉTODO}

Para este estudio fueron seleccionados 72 pacientes con 91 senos maxilares que presentaban indicación de instalación de implantes en el sector posterior; todos los pacientes fueron tratados en la División de Cirugía Oral y Maxilofacial de la Universidad Estatal de Campinas. Fueron incluidos en la investigación pacientes que accedieran voluntariamente y que presentaban documentación clínica completa con radiografías panorámicas preoperatorias y postoperatorias. Fueron excluidos pacientes con patologías

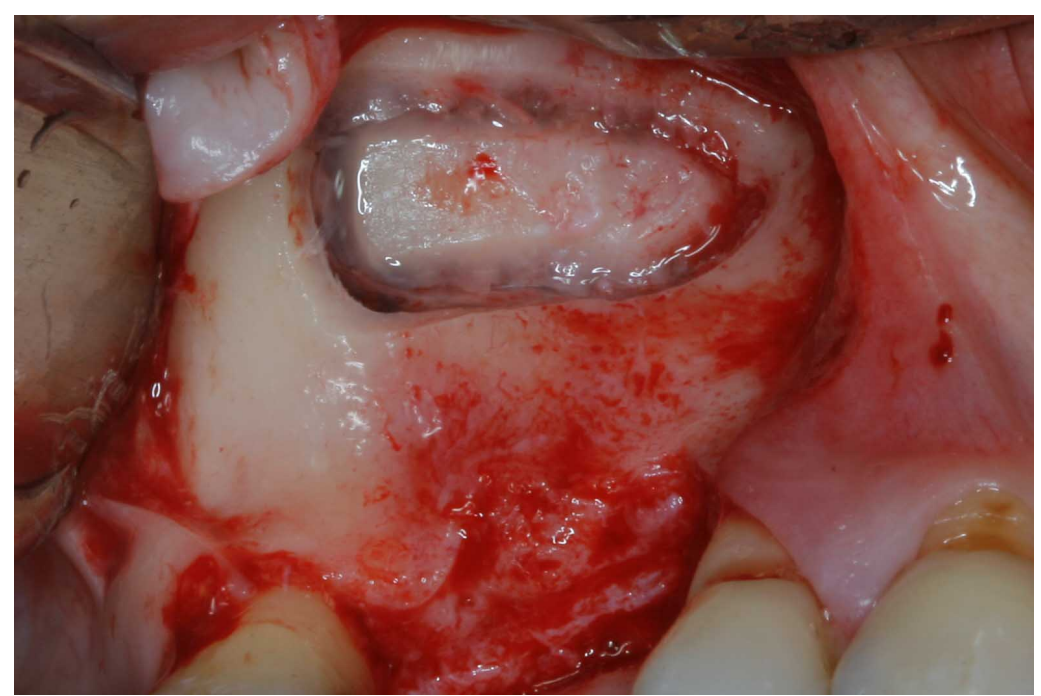

Fig. 1. Técnica quirúrgica de acceso al seno maxilar por ventana lateral mediante una osteotomía en sentidos verticales y horizontales que permite descolar la membrana sinusal de las paredes óseas. sinusales pasadas y patologías respiratorias repetidas; este último grupo de pacientes fue evaluado y tratado por el equipo de otorrinolaringología previo a cualquier intervención quirúrgica sinusal.

Estudio preoperatorio. Todos los pacientes fueron sometidos a estudio mediante radiografía panorámica, en la cual se estableció el sector de instalación de implantes, cuya altura fue medida mediante un pie de metro digital, registrándose en milímetros la distancia del reborde alveolar. La medición fue realizada en tres oportunidades diferentes con diferencia de 5 días entre ellas de forma que el valor promedio obtenido de las tres mediciones fue utilizado como valor final. El valor obtenido fue multiplicado por la constante 0,75 intentando imitar el efecto de la distorsión en la radiografía panorámica. Con este valor, los 91 senos maxilares fueron divididos según la altura ósea mayor o menor que $5 \mathrm{~mm}$ estableciendo así la posibilidad de instalación de implante inmediato o retardado, así como también el tipo de injerto óseo

Procedimiento quirúrgico. Los procedimientos fueron realizados con anestesia local o general dependiendo de las condicionantes del paciente y del procedimiento. Inicialmente ser realizó infiltración del sector a operar mediante anestesia local de lidocaína al $2 \%$ con vasconstrictor (epinefrina 1:100.000). A continuación, con un bisturí número 15 , se realizó un colgajo mucoperióstico que permitiera un acceso adecuado a la pared lateral del seno maxilar; con una fresa esférica montada en motor de baja revolución (25.000 rpm) se realizó una osteotomía horizontal $3 \mathrm{~mm}$ a $5 \mathrm{~mm}$ superior al límite inferior del seno maxilar y una osteotomía vertical $3 \mathrm{~mm}$ a $5 \mathrm{~mm}$ posterior al límite anterior del seno maxilar. A partir de estas osteotomías, se realizó una segunda osteotomía vertical posterior y una osteotomía horizontal superior, formando una ventana de aproximadamente $1 \mathrm{~cm}^{2}$. A continuación se procedió a descolar la membrana sinusal con curetas especiales para este fin, comen- 
zando por la parte inferior y anterior para luego extenderse hacia la región posterior y superior; el decolamiento se realizó hasta tener la suficiente altura para la instalación de implantes; en este momento, la laceración de la membrana sinusal fue registrada y cuando existió se procedió a suturar la membrana a la pared ósea, instalar una membrana absorbible o definitivamente postergar el procedimiento para 3 meses después, esperando la regeneración de la membrana sinusal.

El injerto empleado fue obtenido de rama mandibular, sínfisis mandibular o de cresta iliaca; también fueron utilizados biomateriales como hueso bovino liofilizado, proteína ósea morfogenética y mezclas de estos biomateriales con hueso

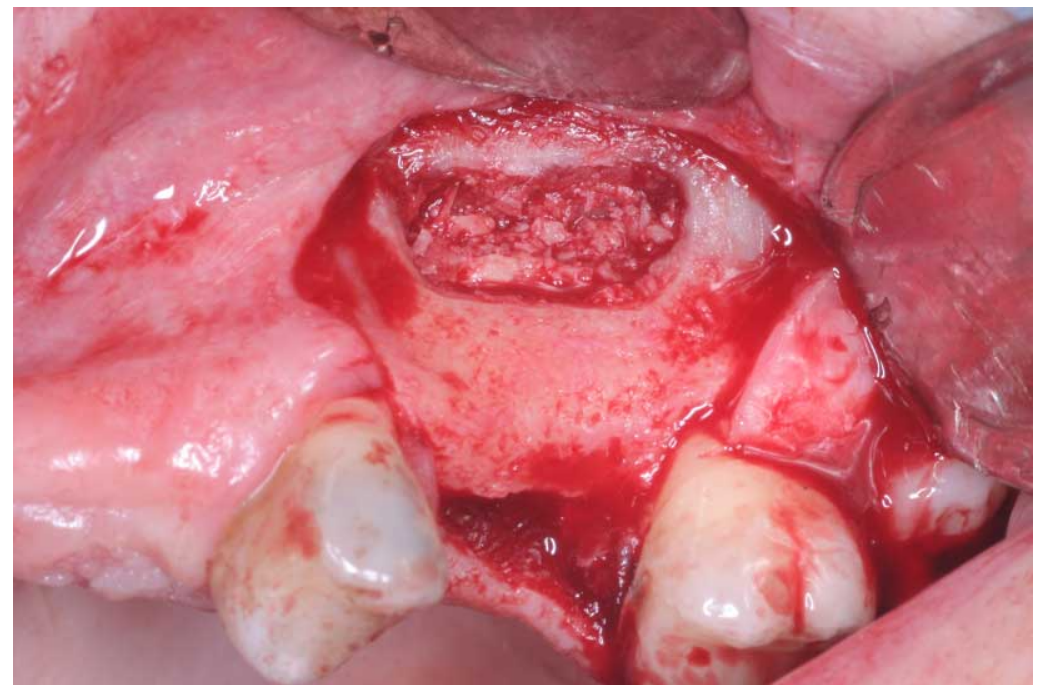

Fig. 2. Relleno óseo intrasinusal con hueso autógeno obtenido de rama mandibular; el hueso es particulado y se aplica al interior de la cavidad realizando una presión moderada hasta cubrir completamente el defecto óseo.

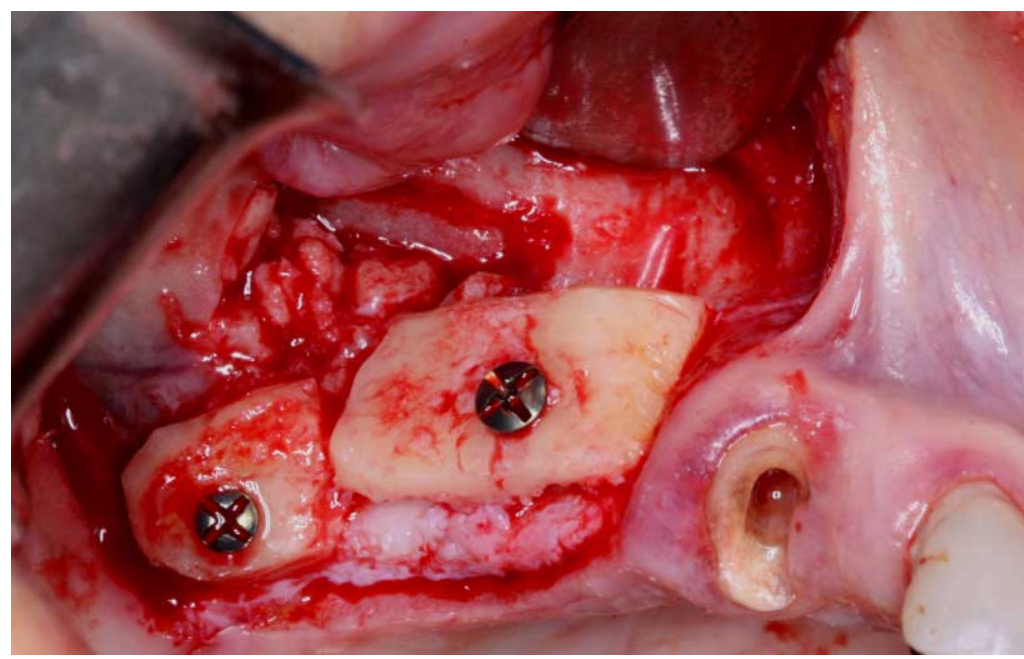

Fig. 3. Instalación del relleno óseo intrasinusal con hueso autógeno (para mejorar la altura del sitio a implantar) y aumento del ancho óseo mediante la instalación de bloques óseos fijados con tornillos de osteosíntesis de titanio del sistema 1.5 . autógeno. La instalación del injerto particulado se desarrolló mediante la aplicación de las partículas óseas con leve compresión hasta cubrir completamente el relleno necesario (Figs. 2 y 3 ). Cuando finalizó esta etapa, se procedió a suturar con sutura absorbible y puntos simples; la terapia farmacológica consistió de amoxicilina $1 \mathrm{~g}$ cada $12 \mathrm{~h}$ vía oral y analgesia mediante AINE. Los pacientes fueron controlados a los 7 y 21 días y finalmente previo a la segunda cirugía de instalación de implante. Se consideraron complicaciones intraoperatorias y postoperatorias la laceración de membrana sinusal, infección, sinusitis y comunicación buco sinusal.

Implantes. Los implantes fueron estudiados en relación al tipo, diseño y su forma de instalación: la instalación inmediata ocurrió cuando se realizó junto al injerto óseo (Figs. 4A y 4B) y la instalación retardada se presento cuando el implante fue instalado luego de al menos cuatro meses desde el momento de instalación del injerto. El éxito de los implantes fue establecido mediante la estabilidad primaria, ausencia de radiolucides perimplantar, ausencia de dolor o infección y optima instalación del tornillo de cicatrización o transmucoso.

Análisis estadístico. Los datos fueron ingresados a una planilla estadística de Microsoft Excel. La relación estadística se aplicó con la prueba test de Fisher considerando el valor de $p<0,05$ para establecer significancia estadística.

\section{RESULTADOS}

Fueron operados 91 senos maxilares correspondientes a 72 pacientes, siendo 53 sujetos del sexo femenino $(73,61 \%)$ y 19 del sexo masculino $(26,39 \%)$; la edad de los sujetos se presentó entre 18 y 66 años con una edad media de 44,56 años; solo 9 sujetos reportaron uso regular de tabaco. En 19 pacientes se realizó la elevación de seno 

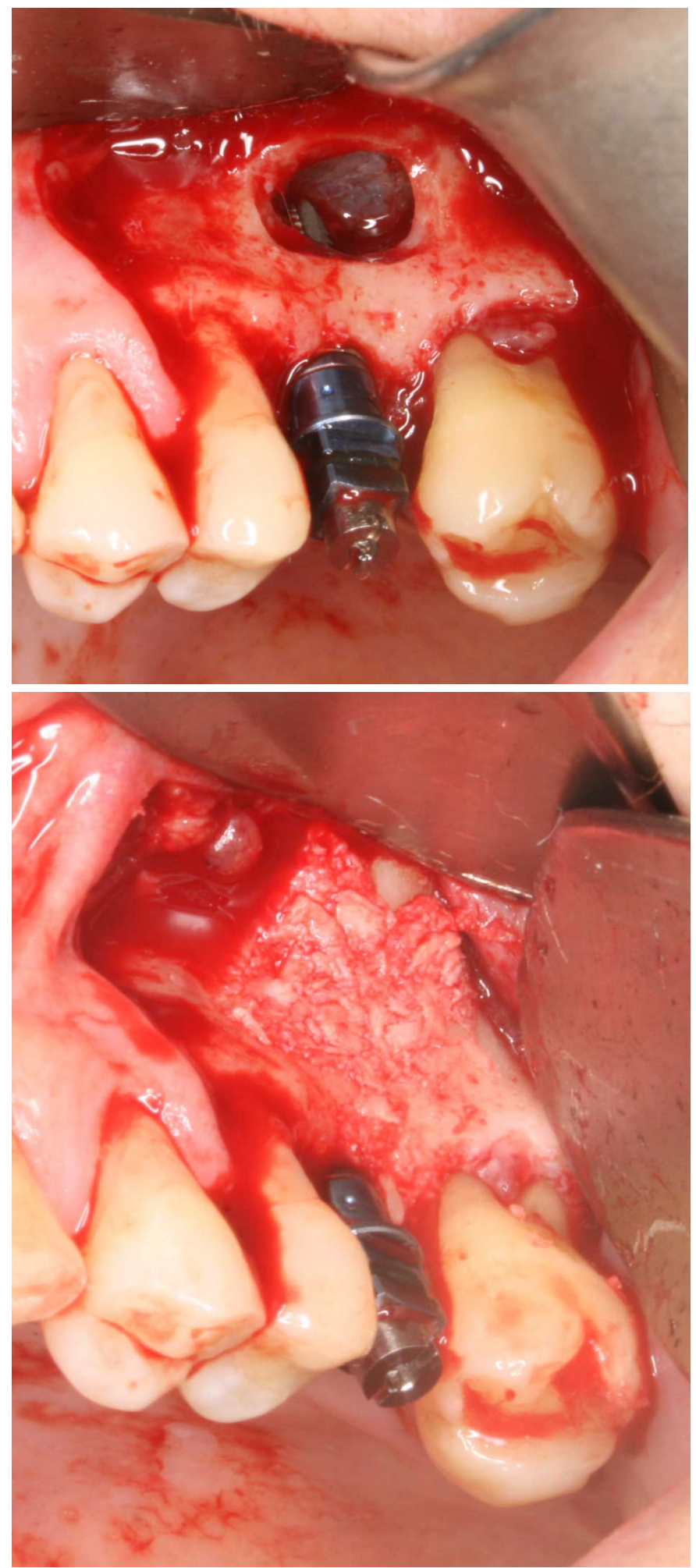

Fig. 4. A. Técnica de elevación del seno maxilar con la instalación del implante de forma inmediata. B. Instalación del relleno óseo intrasinusal cubriendo la totalidad del implante en la región media y apical; el hueso fue obtenido de rama de mandibula. de forma bilateral, mientras que los restantes 53 pacientes fueron operados 31 senos del lado derecho y 22 senos del lado izquierdo. En 80 cirugías fue realizado solo el injerto inlay, mientras que en 11 operaciones se asoció a injerto onlay.

Injerto óseo e implante. En $68(74,7 \%)$ casos fue utilizado hueso autógeno aislado o asociado con algún biomaterial. En 59 casos $(86,8 \%)$ fueron utilizados sitios donantes intrabucales, mientras que en los restantes 9 casos fue utilizado sitio donante extrabucal (hueso proveniente de cresta iliaca uniateral). Complicaciones del sitio donante fueron observadas en 2 casos por dehiscencia de sutura del injerto obtenido de sínfisis mandibular.

La altura ósea previa al tratamiento fue $6,15 \mathrm{~mm} \pm 2,29 \mathrm{~mm}$; en total fueron instalados 101 implantes de diferentes marcas comerciales, de una altura media de $11,93 \mathrm{~mm} \pm 1,58 \mathrm{~mm}$. La Tabla I muestra la distribución de implantes según el reemplazo dentario.

Tabla I. Distribución de los 101 implantes instalados en senos maxilares aumentados según el diente reemplazado.

\begin{tabular}{lcc}
\hline Reemplazo dentario & $\mathbf{n}$ & $\%$ \\
\hline Segundo Molar & 7 & 6,9 \\
Primer Molar & 47 & 46,5 \\
Segundo Premolar & 33 & 32,7 \\
Primer Premolar & 14 & 13,9 \\
Total & 101 & 100 \\
\hline
\end{tabular}

De los 101 implantes, 70 fueron instalados $(69,3 \%)$ en un segundo procedimiento quirúrgico, mientras que los otros 31 fueron instalados de forma inmediata junto a la elevación del seno maxilar con la técnica de ventana lateral. No se observó relación estadística entre el éxito de los implantes instalados en un tiempo quirúrgico o en dos tiempos quirúrgicos $(p>0,05)$.

Al momento de la instalación del conector, en 4 implantes (3,9\% de los implantes) instalados en 3 pacientes, se observo la ausencia de oseointegración, lo que llevo al retiro del implante para posterior instalación en otro tiempo quirúrgi$c o$. No se observo relación estadísticamente significativa en este punto $(p>0,05)$ con la presencia y el tipo de injerto óseo realizado. 
Complicaciones. En 68 operaciones no se observó ningún tipo de complicación (74,7\%). La complicación mas prevalente fue la laceración de membrana sinusal $(18,7 \%)$, presente en 17 operaciones, de las cuales 13 cirugías fueron detenidas y realizadas en un segundo acto operatorio. Los restantes 6 casos de complicaciones correspondieron a infecciones sinusales (3 casos) asociados o no a patología preexistente no diagnosticada, dehiscencia de sutura y pérdida del injerto óseo intrasinusal y la exposición del injerto óseo utilizado para mejorar el ancho alveolar debido a la dehiscencia de la sutura.

\section{DISCUSIÓN}

La técnica utilizada para la elevación del piso de seno maxilar, técnica de ventana lateral, ha sido ampliamente empleada. Con la evolución tecnológica y del conocimiento quirúrgico ha presentado amplias variaciones técnicas con altos índices de éxito, optimizando cada vez mas el tiempo quirúrgico y los resultados postoperatorios (Raja).

Una revisión de estudios clínicos que soporta la reconstrucción con hueso particulado intrasinusal, sostiene que la reconstrucción con autoinjerto presenta mayor formación ósea que cualquier otro injerto aloplástico, homógeno o heterógeno evaluado (Merkx et al., 2003). En este sentido, Watanabe et al. (1999) señalan que en un modelo experimental con injerto autógeno, ya 2 semanas después de realizado el injerto es posible observar la formación de nuevo hueso; en la misma dirección Xiu et al. (2005), utilizando un modelo semejante, señalan que existe una curva de neoformación ósea que es ascendente en las primeras etapas y descendente en las ultimas debido a la remodelación ósea intrasinusal y al estimulo óseo limitado (no se instalaron implantes).

A pesar de que el injerto autógeno es el gold estándar en la reconstrucción, la búsqueda por otros materiales ha llevado a situaciones y conclusiones confusas en la literatura. Por ejemplo, Hallman et al. (2001, 2005) sugieren que el hueso bovino desproteinizado presenta propiedades osteoconductivas, motivo por el cual es posible encontrar hueso inmaduro luego de 6 meses de realizado el injerto óseo intrasinusal lo que llevaría a una mayor pérdida de implantes por la falta de oseointegración. Sin embargo, concluyen que es posible obtener éxito utilizando mezclas de hueso bovino desmineralizado con hueso autógeno. Por otra parte, Garlini et al. (2010) presentan un estudio con el seguimiento radiográfico de implantes instalados en 27 senos maxilares elevados con hydroxiapatita, con una tasa de éxito del $100 \%$. La metodología de trabajo fue con implantes instalados de forma inmediata, de forma que se puede asumir al menos $5 \mathrm{~mm}$ de altura ósea para instalar los implantes; en este tipo de trabajos, el injerto instalado es solo un "ayudante" al recubrimiento del implante y no cumplen un rol importante en las primeras etapas de la oseointegración, ya que esta se produce en el contacto implante-hueso (alveolar o basal remanente en el piso del seno maxilar) y no el injerto aloplástico instalado, de forma que concluir en base a esta investigación que el material aloplástico es factible de ser utilizado en elevaciones de seno maxilar es temerario y en nuestra opinión, imprudente, ya que el estudio se realiza solamente en una técnica determinada (implante inmediato con ventana lateral) y con un análisis radiográfico que demuestra la pérdida de al menos el $30 \%$ del injerto aloplástico instalado $(1,8 \mathrm{~mm}$ de la altura ósea alcanzada). Para esta técnica de implante e injerto óseo inmediato, es completamente viable y con baja morbilidad el uso de injerto autógeno recolectado de sitios próximos al área quirúrgica como el pilar zigomático maxilar (mediante raspado óseo), presentando una alta taza de éxito, además de conseguir la total oseointegración del implante instalado (observado en las imágenes radiográficas) (Johansson et al., 2010)

La potencialidad de la membrana sinusal para la formación ósea es clara y definida. Srouji et al. (2010) establecieron in vitro que células de la membrana sinusal eran capaces de formar hueso; de esta forma, se puede encontrar una justificación al hecho de realizar solo el levantamiento de la membrana sinusal para obtener hueso (Sohn et al. 2010); de hecho, esta también puede ser el motivo del que muchos materiales (a veces llamados biomateriales) presenten altas tazas de éxito en estudios de seguimiento radiográfico. El reporte de Moon et al. (2011) señala también que al instalar implantes en conjunto con sangre venosa periférica es posible observar neoformación ósea próxima al 40\% (estudiado por histomorfometría) en un periodo de 7 meses posteriores a la cirugía; también muestran que los fracasos de los implantes se deberían a la disminuida estabilidad primaria y no al relleno con sangre.

Además del material de relleno, otro tema relevante es el volumen de injerto óseo necesario, lo que muchas veces es poco considerado por el cirujano. 
Jang et al. (2010) sugieren que el injerto instalado debe mantener contacto intimo con el piso y también con la pared medial del seno maxilar para optimizar las propiedades osteoconductivas en la etapa regenerativa; por otra parte, en senos maxilares de mayor volumen se debe optar por injerto autógeno, debido a las propiedades que presenta, superiores a cualquier otro material utilizado.

Respecto del relleno óseo instalado en el seno maxilar, en nuestra muestra el $74,7 \%$ correspondió a injerto autógeno, mientras que en el $25,3 \%$ se utilizaron biomateriales homógenos o xenógenos; en todos ellos se observó un buen rendimiento dada las condiciones clínicas que respetaban los elementos aquí descritos. La investigación de Block et al. (1998), en un seguimiento de 5 y 10 años, demostró el éxito de los implantes rehabilitados en sitios con elevación de seno maxilar, aunque cuando se utilizó mezcla de injerto autógeno y hueso bovino desmineralizado, existió una regresión de la altura ósea observada en radiografías. Nuestros resultados muestran un $96,03 \%$ de estabilidad del implante al momento de la conexión del pilar transmucoso.

La sinusitis, como complicación postoperatoria fue estudiada por Manor et al. (2010) obteniendo una prevalencia del $4 \%$, asociado principalmente a la presencia de sinusitis previa y a la membrana sinusal delgada (observada en el intraoperatorio); la mayoría de los casos se observaron en mujeres aunque no se revelo asociación estadística con el sexo del paciente. Es importante destacar que factores como laceración de la mebrana, sangramiento u otras variables intraoperatorias no fueron asociadas a la sinusitis. Otro elemento importante es que la presencia de pseudoquistes intrasinusales no es una contraindica- ción para la elevación del seno maxilar y no es indicativo de sinusitis maxilar (Mardinger et al., 2007). Timmenga et al. (1997) también presentó un trabajo con 45 pacientes operados para elevación de seno maxilar y una incidencia de 2 casos como sinusitis postoperatoria, señalando que a pesar de presentar cerca de un $30 \%$ de laceraciones de la membrana sinusal en el intraoperatorio, no se observó ninguna relación entre este evento y la sinusitis posterior. Semejantes resultados se presentaron en nuestra muestra con 3 casos de sinusitis postoperatoria, asociados principalmente a patología sinusal previa $(2,97 \%$ de los casos operados) y 3 casos de exposición del injerto asociado a deficiencias en la tecnica de sutura.

En la investigación de Ardekian et al. (2006) la perforación de membrana se observó en 35 pacientes, donde en rebordes remanentes de $3 \mathrm{~mm}$ o menos de altura, el $85 \%$ presentó laceración de membrana y los senos maxilares que presentaban un reborde residual de $6 \mathrm{~mm}$ presentaban solo un $25 \%$ de laceraciones. Se identificó ausencia de significancia estadística al comparar el éxito de implantes instalados en senos maxilares con laceraciones (obliteradas con esponjas de colágeno) y los senos sin laceraciones, siendo cercano al $94 \%$. Elementos como el ancho de la pared ósea del seno maxilar pueden ser relevantes en la técnica quirúrgica. Zijderveld et al. (2008), estudiando 100 cirugías de elevación de seno maxilar evidenciaron la presencia de un $78 \%$ con pared ósea delgada, $4 \%$ con pared gruesa, $48 \%$ con septo intrasinusal y un $11 \%$ de laceraciones de membrana sinusal. Nuestros resultados fueron de $18,7 \%$ de ruptura de membrana sinusal, debido principalmente a la curva de aprendizaje y al tamaño del seno maxilar de mayor volumen.

OLATE, S.; POZZER, L.; LUNA, A. H. B.; MAZONETTO, R.; MORAES, M. \& BARBOSA, J. R. A. Restrospective research in 91 maxilary sinus floor elevation surgery for implant rehabilitation. Int. J. Odontostomat., 6(1):81-88, 2012..

ABSTRACT: Osseous graft for sinus floor elevation has been used for a long time with good results. The aim of this research was to evaluate the operatory and post operatory characteristic of 91 consecutively surgeries for sinus floor elevation. We selected medical records of 72 patient between 18 and 66 years of age for the treatment with local or general anesthesia. Data analysis was realized with a Fisher test with $p<0,05$ for statistical significance. In the $74.7 \%$ autogenous bone with or without biomaterial was used; in $86,8 \%$ intraoral donor site was used. We installed 101 implants with $69.3 \%$ installed in the second surgical time and the other 31 implant installed in the same surgical time with the bone graft; we did not observe any statistical relation in the survival of implants installed in surgical times one or two $(p>0,05)$. We observed the loss of 4 implants without relation with the material used for sinus lift $(p>0,05)$. The more common complication was membrane laceration in $18.7 \%$, and in 13 of 17 cases with this situation the same surgery was performed at a later time. Finally, it can be concluded that the sinus lift is a recognized and safe procedure showing implant stability for adequate osseointegration.

KEY WORDS: maxillary sinus, dental implant, osseous reconstruction. 
OLATE, S.; POZZER, L.; LUNA, A. H. B.; MAZONETTO, R.; MORAES, M. \& BARBOSA, J. R. A. Estudio retrospectivo de 91 cirugías de elevación de seno maxilar para rehabilitación sobre implantes. Int. J. Odontostomat., 6(1):81-88, 2012.

\section{REFERENCIAS BIBLIOGRÁFICAS}

Adell, R.; Lekholm, U.; Rockier, B. \& Branemark, P. I. A 15year study of osseointegrated implants in the treatment of the edentulous jaw. Int. J. Oral Surg., 10(6):387-416, 1981.

Ardkian, L.; Oved-Peleg, E.; Mactei, E. E. \& Peled, M. The clinical significance of sinus membrane perforation during augmentation of the maxillary sinus. J. Oral Maxillofac. Surg., 64(2):277-82, 2006.

Block, M. S.; Kent, J. N.; Kallukaran, F. U.; Thunthy, K. \& Weinberg, R. Bone maintenance 5 to 10 years after sinus grafting. J. Oral Maxillofac. Surg., 56(6):706-14, 1998.

Boyne, P. \& James, R. A. Grafting of the maxillary sinus floor with autogenous marrow and bone. J. Oral Surg., 38(8):613-6, 1980

Breine, U. \& Brånemark, P. I. Reconstruction of alveolar jaw bone. Scand. J. Plast. Reconstr. Surg., 14(1):2348, 1980.

Chiapasco, M.; Zaniboni, M. \& Rimondini, L. Dental implants placed in grafted maxillary sinuses: A retrospective analysis of clinical outcome according to the initial clinical situation and a proposal of defect classification. Clin. Oral Implant Res., 19(4):416-28, 2008.

Emmerich, D.; Att, W. \& Stappert, C. Sinus floor elevation using osteotomes: a systematic review and metaanalysis. J. Periodontol., 76(8):1237-51, 2005.

Garlini, G.; Redemagni, M.; Donini, M. \& Maiorana, C. Maxillary sinus elevation with an alloplastic material and implants: 11 years of clinical and radiologic follow up. J. Oral Maxillofac. Surg., 68(5):1152-7, 2010.

Griffin, T. J. \& Cheung, W. S. The use of short, wide implants in posterior áreas with reduced bone height: $A$ retrospective investigation. J. Prosthet. Dent., 92(2):139-44, 2004

Hallman, M.; Sennerby, L.; Zetterqvist, S. \& Lundgren, S. A 3-year prospective follow-up study of implant-supported fixed prostheses in patients subjected to maxillary sinus floor augmentation with a 80:20 mixture of deproteinized bovine bone and autogenous bone. Clinical, radiographic and resonance frequency analysis. Int. $J$. Oral Maxillofac. Surg., 34(3):273-80, 2005.

Hallman, M.; Cederlund, A.; Lindskog, S.; Lundgren, S. \& Sennerby, L. A clinical histologic study of bovine hydroxyapatite in combination with autogenous bone and fibrina glue for maxillary sinus floor augmentation. Results after 6-8 months of healing. Clin. Oral Impl., 12(2):13543, 2001
Ivanoff, C. J.; Gröndahl, K.; Sennerby, L.; Bergström, C. \& Lekholm, U. Influence of variations in implant diameters: a 3- to 5-year retrospective clinical report. Int. J. Oral \& Maxillofac. Impl., 14(2):173-80, 1999.

Jang, H. Y.; Kim, H. C.; Lee, S. C. \& Lee, J. Y. Choice of graft material in relation to maxillary sinus width in internal sinus floor augmentation. J. Oral Maxillofac. Surg., 68(8):185968, 2010.

Jensen, J.; Sindet-Pedersen, S. \& Oliver, A. J. Varying treatment strategies for reconstruction of maxillary atrophy with implants: results in 98 patients. J. Oral Maxillofac. Surg., 52(3):210-6, 1994.

Johansson, L.; Isaksson, S.; Lindh, C.; Becktor, J. \& Sennerby, L. Maxillary sinus floor augmentation and simultaneous implant placement using locally harvested autogenous bone chips and bone debrids: a prospective clinical study. J. Oral Maxillofac. Surg., 68(4):837-44, 2010.

Kluppel, L. E.; Santos, S. E.; Olate, S.; Freire Filho, F. W.; Moreira, R. W. \& de Moraes, M. Implant migration into maxillay sinus: description of two asymptomatic cases. Oral Maxillofac. Surg., 14(1):63-6, 2010.

Lekholm, U.; Wannfors, K.; Isaksson, S. \& Adielsson, B. Oral implant in combination with bone grafts: A 3-year retrospective multicentre study using the Bränemark implant system. Int. J. Oral Maxillofac. Surg., 28(3):1817, 1999.

Manor, Y.; Mardinger, O.; Bietlitum, I.; Nashef, A.; Nissan, J. \& Chaushu, G. Late signs and symtoms of maxillary sinusitis after sinus augmentation. Oral Surg. Oral Med. Oral Pathol. Oral Radiol. Endod., 110(1):e1-4, 2010.

Mardinger, O.; Manor, I.; Mijiritsky, E. \& Hirshberg, A. Maxillary sinus augmentation in the presence of antral pseudocyst: a clinical approach. Oral Surg. Oral Med. Oral Pathol. Oral Radiol. Endod., 103(2):180-4, 2007.

Misch, C. M. Comparison of intraoral donor sites for onlay grafting prior to implant placement. Int. J. Oral Maxillofac. Implants, 12(6):767-76, 1997.

Merkx, M. A.; Maltha, J. C. \& Stoelinga, P. J. Assessment of the value of anorganic bone additives in sinus floor augmentation: a review of clinical reports. Int. J. Oral Maxillofac. Surg., 32(1):1-6, 2003.

Moon, J. W.; Sohm, D. S.; Heo, J. U.; Shin, H. I. \& Jung, J. K. New bone formation in the maxillary sinus using peripheral venous blood alone. J. Oral Maxillofac. Surg., 69(9):2357, 2011. 
OLATE, S.; POZZER, L.; LUNA, A. H. B.; MAZONETTO, R.; MORAES, M. \& BARBOSA, J. R. A. Estudio retrospectivo de 91 cirugías de elevación de seno maxilar para rehabilitación sobre implantes. Int. J. Odontostomat., 6(1):81-88, 2012.

Olate, S.; Lyrio, M. C.; de Moraes, M.; Mazzonetto, R. \& Moreira, R. W. Influence of diameter and length of implant on early dental implant failure. J. Oral Maxillofac. Surg., 68(2):414-9, 2010.

Raja, S. V. Management of the posterior maxilla with sinus lift: review of techniques. J. Oral Maxillofac. Surg., 67(8):1730-4, 2009.

Sharan, A. \& Madjar, D. Maxillary sinus pneumatization following extractions: a radiographic study. Int. J. Oral Maxillofac. Implants, 23(1):48-56, 2008.

Sohn, D. S.; Moon, J. W.; Moon, K. N.; Cho, S. C. \& Kang, P. S. New bone formation in the maxillary sinus using only absorbable gelatin sponge. J. Oral Maxillofac. Surg., 68(6):1327-33, 2010.

Srouji, S.; Ben-David, D.; Lotan, R.; Riminucci, M.; Livne, E. \& Blanco, P. The innate osteogenic potential of the maxillary sinus (schneiderian) membrane: an ectopic tissue transplant model simulating sinus lifting. Int. J. Oral Maxillofac. Surg., 39(8):793-801, 2010.

Tatum, O. H. Maxillary and sinus implant reconstruction. Dent. Clin. North Am., 30:207-29, 1986.

Tiwana, P. S.; Kushner, G. M. \& Haug, R. H. Maxillary Sinus Augmentation. Dent. Clin. North Am., 50(3):409-24, 2006.

Timmenga, N. M.; Raghoebar, G. M.; Boering, G. \& van Weissenbruch, R. Maxillary sinus function after sinus lifts for the insertion of dental implants. J. Oral Maxillofac. Surg., 55(9):936-9, 1997.

Ulm, C.; Kneissel, M.; Schedle, A.; Solar, P.; Matejka, M.; Schneider, B. \& Donath, K. Characteristic features of trabecular bone in edentulous maxillae. Clin. Oral Implants Res., 10(6):459-67, 1999.

Viña-Almunia, J.; Peñarrocha-Diago, M. A. \& PeñarrochaDiago, M. Influence of perforation of the sinus membrane on the survival rate of implants placed after direct sinus lift. Literature update. Med. Oral Patol. Oral Cir. Bucal, 14(3):E133-6, 2009.

Watanabe, K.; Niimi, A. \& Ueda, M. Autogenous bone grafts in the rabbit maxillary sinus. Oral Surg. Oral Med. Oral Pathol. Oral Radiol. Endod., 88(1):26-32, 1999.

Wiltfang, J.; Schultze-Mosgau, S.; Nkenke, E.; Thorwarth, M.; Neukam, F. W. \& Schlegel, K. A. Onlay augmentation versus sinus lift procedure in the treatment of the severely resorbed maxilla: A 5-year comparative longitudinal study. Int. J. Oral Maxillofac. Surg., 34(8):885-9, 2005.
Xiu, H.; Shimizu, Y. \& Ooya, K. Histomorphometric study of the stability of newly formed bone after elevation of the floor of the maxillary sinus. Br. J. Oral Maxillofac. Surg., 43(6):493-9, 2005.

Zijderveld, S. A.; van den Bergh, J. P.; Schulten, E. A. \& ten Bruggenkate, C. M. Anatomical and surgical findings and complications in 100 consecutive maxillary sinus floor elevation procedures. J. Oral Maxillofac. Surg., 66(7):1426-38, 2008.

Dirección para correspondencia:

Prof. Dr. Jose Ricardo de Albergaria Barbosa

Av. Limeira 901

Caixa Postal 52

CEP 13414-903

Piracicaba - SP

BRASIL

Email: barbosa@fop.unicamp.br -solate@ufro.cl

Recibido : 18-11-2011

Aceptado: 27-12-2011 